\title{
Mentalisierung und Neuropsychoanalyse
}

\author{
Regula Schiess, Max Haas (Zürich und Basel)
}

Zusammenfassung: In der «Philosophie des Geistes» genannten Forschungsrichtung werden u.a. mentale Zustände (mental states) behandelt. Davon abgeleitet ist die «Mentalisierung» genannte Forschungsrichtung. Sie beschäftigt sich mit der «reflexiven Funktion» im Sinne, «eigenes und fremdes Verhalten als durch mentale Zustände (Wünsche, Phantasien, Ängste) motiviert zu verstehen» (Peter Potthoff [2008:86]). In einer entwicklungspsychologischen Lesart geht es dabei um die Entwicklung des Selbst. Für die Theoriebildung ist es interessant, die vielleicht primitiv anmutenden, in sich aber gut begründeten Ansätze der Neurowissenschaften, insbesondere der von Rolf Pfeifer vorangetriebenen Roboterforschung heranzuziehen, um zu prüfen, inwiefern diese die reflexive Mentalisierungsfunktion von Therapeuten stützen kann. Im Hintergrund stellt sich die Frage, wie weit die Metapsychologie eine Mentalisierungsfunktion hat. Die vorliegende Arbeit liefert keine Antwort, sondern stellt unterschiedlich motivierte Ansätze vor.

Schlüsselwörter: Mentalisierung, Robotik, Neuropsychoanalyse, Anthropomorphismus, Kategorienfehler, Gegenübertragung, Embodiment, Metapsychologie

\section{$1 \quad$ Einleitendes}

Wir möchten den Begriff «Mentalisierung» nicht aufgreifen als jenen magischen Drehpunkt einer modernen Psychotherapietechnik, die sich wie eine Art Hochleistungssport zur Behandlung von diagnostisch genau abgegrenzten Patientengruppen darstellt. Auch glauben wir nicht daran, dass durch Mentalisierungsarbeit die Affektregulierung von ganzen Kulturen verbessert werden kann (Bolm [2009: 150]). Wir berufen uns hier vielmehr auf die Kompetenz, den menschlichen Austausch zu reflektieren. Oder praxisnahe und althergebracht ausgedrückt: Wir arbeiten mentalisierungsorientiert, wenn wir das Containing beachten, dem Patienten Halt und Raum geben, um schwierig auszuhaltende Emotionen ins Bewusstsein zu holen, zu benennen und zu reflektieren. Wie der Patient dieses Therapieangebot beantwortet, ergibt dann den Fahrplan für den therapeutischen Prozess und die Beziehungsentwicklung. Wir beschränken uns hier 
auf die Potthoff'sche Version von Mentalisierung als einer reflexiven Funktion, die «eigenes und fremdes Verhalten als durch mentale Zustände (Wünsche, Phantasien, Ängste) motiviert» versteht (Potthoff [2008:86]). Dieser Begriff von Mentalisierung soll im Zusammenhang mit anderen Forschungsrichtungen beleuchtet werden.

Wir Psychoanalytiker sollten trotz Auseinandersetzungen mit und gewissen Anpassungen an die heute geforderten Standards der evidenzbasierten Medizin und an die Ökonomisierung aller menschlichen Kontakte im Gesundheitswesen nicht vergessen, dass die Natur des Menschen nach wie vor Rätsel aufgibt. Und es geht darum, zusammen mit andern Wissenschaftlern verschiedener Disziplinen am Erkenntnisprozess mitzuwirken. Das bedeutet für Psychoanalytiker, bei der Metapsychologie anzusetzen. Sie ist unsere theoretische Erkenntnisebene. Solange wir uns nicht mehr an die metapsychologischen Fragen herantrauen, bleiben wir reine Kliniker und versuchen, im klinischen Rahmen zu verstehen und zu erklären. Wir wissen, dass unsere Therapien eine Wirkung haben, auch wenn sich die Erklärungsgrundlagen, auf denen sie beruhen, später als falsch erweisen. Aber wir müssen zugleich zur Kenntnis nehmen, dass wir als reine Kliniker der Gesundheitspolitik in besonderem Masse ausgeliefert sind.

Befassen wir uns dagegen mit dem Psychischen nicht mehr direkt aus klinischem Interesse, sondern schauen beispielsweise, wie andere psychologische Schulen und an die Psychoanalyse angrenzende Wissenschaftszweige psychische Vorgänge und das spezifisch Menschliche erfassen, stellt sich die Frage, wie die Phänomene geordnet und benannt werden. Es lässt sich gut nachvollziehen, dass Ernst Pöppel - mit seiner über dreissigjährigen Lehrerfahrung in medizinischer Psychologie ein Experte auf dem Gebiet - nach wie vor eine «übersichtliche Landkarte von psychischen Phänomenen» vermisst. Er meint, in Anlehnung an die Modellbildung in axiomatisch-deduktiven Systemen im Sinne Rudolf Carnaps, die heuristisch etablierten Modelle in der Psychologie seien in der Regel dann am besten, «wenn man sich auf eine beschränkte Auswahl psychischer Phänomene bezieht, die sich von vornherein für eine explizite Modellierung anbieten, wie es beim psychophysischen Modell der Fall ist.» (Pöppel/Ruhnau [2010, Abs. 9]). Es ist allerdings genau dieser Reduktionismus, der die akademische Psychologie in der Regel so langweilig macht. Andererseits ist die Verständigung über das, was sinnvoll gemeint ist, selbst unter Psychoanalytikern oft schwierig zu eruieren, obgleich in diesem Kreis die klinische Theoriebildung einem einheitlichen Zweck dient und das Praxisfeld mit dem Therapiesetting und der analytischen Haltung zusätzlich vereinheitlichend wirkt. 
Trotz enormer Orientierungs- und Verständigungsschwierigkeiten hat sich eine extra-klinische psychoanalytische Forschung und Theoriebildung entwickelt. Letztere hat in der psychoanalytischen Bewegung als concept research einen wichtigen Platz. Wir zitieren Marianne Leuzinger-Bohleber und Tamara Fischmann (2006: Abstract), welche im Namen des Research Subcommittee for conceptual research of the International psychoanalytical association zusammenfassend festhalten:

"The development of psychoanalysis as a science and clinical practice has always relied heavily on various forms of conceptual research. Thus, conceptual research has clarified, formulated and reformulated psychoanalytic concepts permitting to better shape the findings emerging in the clinical setting. By enhancing clarity and explicitness in concept usage it has facilitated the integration of existing psychoanalytic thinking as well as the development of new ways of looking at clinical and extraclinical data. Moreover, it has offered conceptual bridges to neighbouring disciplines particularly interested in psychoanalysis, e.g. philosophy, sociology, aesthetics, history of art and literature, and more recently cognitive science/neuroscience. In the present phase of psychoanalytic pluralism, of worldwide scientific communication among psychoanalysts irrespective of language differences and furthermore of an intensifying dialogue with other disciplines, the relevance of conceptual research is steadily increasing."

Dem entsprechend bringen wir in Abschnitt 2 mit Don Tuckers Arbeit ein Beispiel sowohl für ein differenziertes interdisziplinäres Aufgreifen metapsychologischer Grundbegriffe als auch für ein der Psychoanalyse ähnliches Erfassen psychischer Funktionen, jedoch im Gewand einer anderen Begrifflichkeit. Dann versuchen wir in Abschnitt 3 aufzuzeigen, wie philosophisches Nachdenken helfen kann, «Kategoriengewohnheiten durch Kategoriendisziplin zu ersetzen» (Lorenz [1995:370b]) und so Fehler aufzudecken. Im Abschnitt 4 gehen wir auf die spannenden Möglichkeiten ein, die sich der Konzeptforschung durch die embodied cognitive science bietet.

\section{Don Tucker's Cognitive Neuroscience}

Anfangs der 90er Jahre begann sich die Bewegung der Neuropsychoanalyse mit Unterstützung der New York Academy of Sciences auszubreiten. Aus Anlass des 100jährigen Entstehens von Freuds Entwurf einer Psychologie (1895) wurde 
eine Tagung mit verschiedenen hochdotierten Rednern - Neurologen, Neuropsychologen, Psychoanalytikern - abgehalten (Bilder, LeFever [1998]). Zu ihnen gehörten auch die beiden Vertreter der kognitiven Neurowissenschaften Don Tucker und Phan Luu (1998). Ihre Arbeit zum Thema des psychoanalytischen Besetzungsbegriffs aus neurowissenschaftlicher Sicht beginnt mit der Frage, wie Neuronen Erfahrungen repräsentieren und Verhalten adaptiv organisieren können. Und es folgt eine Auseinandersetzung mit Freuds Vorstellung, dass das Erinnern die neuronalen Mechanismen verändert, während beim Wahrnehmen die Neuronen gleichbleiben. Die Autoren führen mit neurowissenschaftlichen Methodologien erlangte Forschungsresultate ins Feld, um die corticolimbischen Interaktionen möglichst genau beschreiben zu können, die dem Zusammenhang zwischen Fühlen,Wünschen und Erinnern zugrundeliegen. Sie weisen dann weiter daraufhin, dass Freud sich getäuscht habe, als er die neuronalen Netzwerke, die zuständig sind für Wahrnehmen und für Erinnern, voneinander getrennt habe. Beide psychischen Funktionen beträfen dieselben cortikalen Bahnen. Freuds Hinweis auf die Spannung zwischen Wahrnehmungsstabilität und mnestischer Plastizität sei jedoch auch heute noch relevant als Stabilitäts-/Plastizitäts-Dilemma. Die Erfahrungen des Individuums zeigten sich in seinen durch Motive geleiteten Erwartungen und in den Wahrnehmungen, die durch Erwartungen motiviert seien sowie in seiner Fähigkeit, in jeder Lebenslage Erinnerungen an ähnliche Situationen zu mobilisieren und in die Affektregulierung und Handlungsplanung einzubeziehen. Dabei sind Motive bei Tucker alle über den Hypothalamus vermittelten Triebregungen aus den beiden Triebkategorien: Selbsterhaltungstriebe und Sexualtrieb. Motiviert ist das Verhalten insofern, als es die in die Erfahrungen und Lernprozesse eingegangenen Triebe mehr oder weniger deutlich erkennen lässt. (Siehe zur Formalisierung dieser zentralen Aspekte der Freud'schen Metapsychologie die Arbeit von Schmidt-Hellerau [2003]).

Tucker entwickelt später (2007) eine Art von Multilog ähnlich jenem von Freud bis etwa 1900 unter Einbezug von a) den Grundbegriffen und Mechanismen des psychischen Apparats, b) vergleichender Neuroanatomie, c) Evolutionstheorie, d) experimenteller Psychologie und Tierforschung, e) Neurophysiologie, f) Informatik und Kybernetik. Dies erlaubt ihm der Frage nachzugehen, was denn genau auf der Ebene des Hirns geschieht, bzw. wie man es sich denken könnte, was geschieht, wenn ein Mensch im Alltag Erfahrungen macht, erschrickt, in Konflikte gerät oder unter Zeitdruck Lösungen suchen muss. Tucker liefert eine für Psychoanalytiker wertvolle Zusammenstellung von zeitgenössischem Wissen zum alltäglichen psychologischen Funktionieren. Damit beinhaltet seine Psychologie eine für einen 
Akademiker ungewöhnlich breite Auswahl an Phänomenen. Weder die Triebe noch das verkörperte emotionale und andere Wissen, ob unbewusst oder bewusst, werden ausgelassen. Auch scheut er sich nicht, nach gründlicher Abwägung, Hypothesen über die Funktionsabläufe im Hirn aufzustellen. Eines seiner Hauptkonzepte ist dabei die vertikale Integration der verschiedenen Hirnebenen vom Hirnstamm bis zum Cortex, womit er sich in die Tradition Alexander Lurijas stellt. Eine weitere zentrale Überlegung ist, dass eine bestimmte Verknüpfungsarchitektur eines Hirnbereichs mit einer bestimmten Funktion dieses Bereichs zusammenhängen muss. Er geht von der pragmatischen Haltung aus, dass «die neuronalen Strukturen [...] nicht einfach passive Begrenzungen der Erfahrung (sind). Sie sind die Vehikel mittels denen (man) Kontrolle über sich gewinnt» (2006:184f.).

Mentalisierung in Tucker'schen Worten würde eine «zweigleisige Interaktion zwischen abstrakten, kognitiven repräsentationalen Fähigkeiten des Cortex und der elementaren Motivkontrolle [qua Triebkontrolle, R.S.] der subcorticalen Schaltkreise» beinhalten. Zweigleisig ist diese Interaktion, soweit bottomupund topdown- Aktivierungen impliziert sind. Das zeige sich auf der Ebene des Erlebens in einer Durchmischung von Gefühlen (wie zum Beispiel einer Angst als viszeraler Reaktion auf eine als gefährlich beurteilte Situation) und Ideen (wie zum Beispiel dem Antizipieren von missbilligenden Blicken als semantischer Resonanz usw.). Gelingt diese Art von innerem Dialog, ergibt sich ein stabiler mentaler Bezugsrahmen, der den sense of agency - die Handlungsbereitschaft - so weit stärkt, dass sich Vertrauen in die Selbstkontrolle ausbreitet; dies ausgehend von der limbischen Basis der Frontallappennetzwerke. Es gelte, die «Gefühle genug lange aushalten (zu können) und so die optimalen Motivmuster in [...] die Erfahrung» einzubringen (2006:185). Dabei sehen wir unter 4., wie die embodied cognitive science versucht, diesen Tucker'schen Erfahrungsbegriff ein Stück weit zu operationalisieren, indem der sich verhaltende Organismus örtlich und zeitlich situiert wird.

\section{$3 \quad$ Philosophisches Zwischenspiel}

Eine neuropsychoanalytische Orientierung impliziert ein Zusammentreffen von (herkömmlicher) Psychoanalyse mit den Neurowissenschaften. Tritt man, wie das heute üblich geworden ist, für eine Brücke und nicht für einen Gegensatz zwischen Natur- und Geisteswissenschaften ein, bietet Ludwig Wittgenstein eine Leitidee. Bei ihm heisst es einmal: «Wer in ein fremdes Land kommt, wird manchmal die Sprache der Einheimischen durch hinweisende Erklärungen lernen, die sie ihm geben; und er wird die Deutung dieser Erklärungen oft raten müssen und manch- 
mal richtig, manchmal falsch raten.» (Philosophische Untersuchungen $\S 32$ ). Für uns Kliniker ist der Patient ein fremdes Land; aufgrund unserer Ausbildung, unserer Erfahrung, unserer Intuition verfügen wir über Ratemodelle qua «Mechanismen» und «Entwicklungstheoriemodellen», um uns mit dem Patienten zusammen im fremden Land zurechtzufinden. Wir versuchen, im Sinne eines groben Rasters zwei mögliche Grundlagen solcher Modelle zu skizzieren.

Erstens: Die Philosophie ist auch zuständig für die Frage, was sprachlich korrekte Sätze sind. Für uns Kliniker ist das ein wichtiges Problem. Denn in Therapie, Supervision, Intervision und Vorträgen äussern wir uns sprachlich und inszenieren dabei alle Mitteilungen auch körperlich. Zudem ergänzen wir unsere mündlichen Äusserungen der Situation entsprechend: wir fügen an, modifizieren Gesagtes oder erklären die Reaktion unseres Gegenübers entsprechend. Schreiben wir aber Aufsätze und Bücher, ist das geschriebene Wort der einzige Stellvertreter; die körper- und situationszentrierte Klärung des Gemeinten entfällt. Daraus ergibt sich ein Bedarf an sprachphilosophischer Klärung für den Fall des Schreibens, wie sich aus der vom englischen Philosophen Gilbert Ryle angezettelten Diskussion um den sogenannten Kategorienfehler ergibt. Abstrakt ausgedrückt liegt ein Kategorienfehler dann vor, wenn ein Ausdruck «eines bestimmten logischen Typs ... an solchen Leerstellen einer Aussageform» eingesetzt wird, «die nur für Ausdrücke anderer logischer Typen eine sinnvolle Aussage» ist (Lorenz [1995:370b]). So lässt sich im Muster «Gestern traf ich X in Zürich» X durch «Hans» ersetzen: «gestern traf ich Hans in Zürich» ist korrekt, aber der Satz «gestern traf ich den durchschnittlichen Steuerzahler in Zürich» ist falsch; denn «Hans» und «durchschnittlicher Steuerzahler» gehören zwei verschiedenen Kategorien an. Diese Problematik scheint zunächst nur sprachphilosophisch relevant. Allerdings führten die daraus entstehenden Diskussionen zu Klärungen von mentalen Ausdrücken (Beckermann [2001:76-79]), deren Konsequenzen auch die Robotik (Pfeifer/Bongard [1999: 503-505]) erreichten.

Marianne Leuzinger-Bohleber (2009:152-159) zeigt im Anschluss an Pfeifer und Scheier (1996), welche Nachteile das Gedächtnis im Sinne des SpeicherModells für die Theoriebildung hat und dass die Speicher-Metapher in der akademischen Psychologie zu unproduktiven und schwerfälligen Fragestellungen führt und Erkenntnisfortschritte verhindert. Es ist uns Psychoanalytikern bekannt, dass sich wichtige lebensgeschichtliche Ereignisse des Patienten zuerst in einer Gegenübertragungsreaktion des Therapeuten zeigen können. Die dem Patienten nicht bewusste Erinnerung «kommt» in den Körper des Therapeuten, welcher bei sachgemässem Lesen des eigenen Körpers erkennen kann, dass es sich um von der 
Empathie mit dem Patienten ausgelöste Empfindungen und Gefühlszustände handelt. Diese können auf implizite Wahrnehmungsprozesse zurückgeführt werden. Leuzinger-Bohleber (2009: 160 f.) beschreibt, wie im Verlaufe einer Behandlung ihre «Wahrnehmungen feinster sensomotorischer Signale des Analysanden und die dadurch ausgelösten inneren Koordinations- und Kategorisierungsprozesse sukzessiv differenzierter» wurden und «daher die damit verbundenen, intensiven affektiven und körperlichen Empfindungen» evozierten. So habe sie schliesslich analoge Körperempfindungen wie der Analysand selbst entwickelt: Spannungen, Unwohlsein, Magenkrämpfe. Der Körper des Patienten erinnert sich ohne Bewusstsein so wie sich die Hände des Pianisten einer Chopin-Etüde erinnern.

Das Gedächtnis als Speicher zu denken ist das, was die Philosophen fehlerhafte Kategoriengewohnheit bzw. Kategorienfehler nennen. Das trifft auch auf das Erinnern, gedacht als körperlich verankerter, neuroplastischer Prozess zu. Denn es geht um ein Geschehen, das in einer ersten Interaktion mit der Umgebung beginnt und in einer zweiten, ähnlichen zum Vorschein kommt. Und über die zugrundeliegenden Mechanismen, welche die tatsächliche Verbindung zu Stande bringen zwischen beobachtbarem Verhalten - hier qua Erinnern - und Hirnaktivierung, gibt es noch keine umfassende Kenntnis. Letztlich könne dieser Zusammenhang nur erschlossen werden, wenn ein in einer konkreten Umgebung situierter und in Echtzeit agierender Organismus erforscht werde (Pfeifer/Scheier [1999:531f.]).

Zweitens: Seit Aristoteles gilt die Frage nach den Intentionen menschlichen Handelns (bei Tucker motiviertes Verhalten genannt). Daraus ergeben sich unterschiedliche Schlüsse. Man kann, wie das Roy Schafer (1982) versucht hat, der Metapsychologie Freuds ein handlungstheoretisches Fundament geben. Oder man bezieht sich auf die philosophischen Grundlagen in handlungstheoretischen Ansätzen seit Aristoteles, wie sie etwa Georg Meggle (1977) und Ansgar Beckermann (1977) in Form einer Anthologie vorstellen. Solche Interessen lassen sich mühelos auf die Robotik als Teilbereich der neuropsychoanalytischen Arbeit ausweiten. Roboter handeln und wir sind, wie Pfeifer/Bongard (2006) immer wieder betonen, in Gefahr, solche Handlungen anthropozentrisch auszulegen. Daher werden Roboter für uns sehr interessant, wenn sie dank einfachster neuronaler Netze Inputs lernend verarbeiten und damit - etwa in der Biorobotik - Annahmen über Handlungsintentionen von Tieren erlauben (siehe unten das Referat zum Roboter «Sahabot»).

Anthropomorphismen sind demnach nicht einfach Fehldeutungen. Wir können sie auch als Heurismen verstehen, die zu Überprüfungen und dann zu Korrekturen führen. Das gesamte Gebiet der Neurowissenschaften und damit, 
wie sich zeigen wird, auch der Robotik liefert nicht einfach neue Erkenntnisse, sondern vor allem Ansätze, Gewohntes zu hinterfragen und gewinnbringend Anthropomorphismen zu analysieren.

\section{$4 \quad$ Mentalisierung. Robotik}

Die Aufhebung der Opposition zwischen Geistes- und Naturwissenschaft in der Philosophie des Geistes einerseits (vgl. Pauen/Roth [2001]), die ganz praxisbetonten Anliegen psychoanalytischer Theoriebildung andererseits empfehlen es, auch einmal einen Brückenschlag zu versuchen und zwar den zwischen Theorien zur Mentalisierung und den Überlegungen zur Robotik. Was impliziert das Vorgehen?

In Anlehnung an Fonagy bedeutet Mentalisieren nach T. Bolm (2009: 29-30) «äusserlich wahrnehmbares Verhalten in Zusammenhang mit inneren, «mentalen` Zuständen und Vorgängen zu erleben und zu verstehen und umgekehrt. Bei dieser inneren Realität handelt es sich z. B. um Gefühle, Gedanken, Bedürfnisse, Wünsche, Begründungen, Bedeutungen und ganz persönliche Lebenserfahrung. [...] Mentalisieren bedeutet nicht nur zu verstehen, was in uns und anderen Menschen vor sich geht (Verständnis erster Ordnung), sondern auch die Art und Weise zu begreifen, wie wir und andere dies tun (Verständnis zweiter Ordnung, Metakognition).» Dieser Sicht entspricht die Feststellung, dass «unser aller Realitätserleben in einem Zwischenbereich zwischen Ausseneinflüssen und eigener Schöpfung angesiedelt» ist. «Die intrapsychische Fähigkeit zum spielerischen Umgang mit innerer und äusserer Realität geht einher mit einem inneren, spielerischen «Möglichkeitsraum» im Sinne von Winnicott (1971).» Das Realitätserleben ist demnach individuell verschieden und situationsabhängig. Realität dagegen ist 〈pluralistisch〉 und wird gemeinsam mit anderen Menschen «geschaffen»».

Dieser Auffassung von menschlicher «Realwelt» soll im Folgenden, ausgehend von Pfeifer/Bongard (2006) die physical realworld der Robotik gegenübergestellt werden. Beide Kategorien von agents - Roboter und Menschen - entwickeln ihr Verhalten in der physikalischen Umwelt, mit der sie interagieren. Dabei ist «Mensch und Kultur» die wichtigste Umweltkomponente des Menschen.

Der fachliche Umgang mit Robotik ist weit mehr als das gelegentliche Programmieren eines Computers zum Zwecke einer Simulation. «... building robots requires engineering expertise which is typically not present in computer science laboratoires; it is messy, you have to get your hands dirty, which is something that, in the age of information technology, many people strongly dislike». (Pfeifer/Bongard [2006:36]). Als Analytiker wenden wir uns demnach einem Gebiet zu, über das 
wir uns informieren lassen, da wir zur selbständigen Arbeit gar nicht fähig sind. Nehmen wir nun bestimmte Leistungen von Robotern zur Kenntnis, haben wir keine regulierende eigene Praxis, die den fachgerechten Umgang mit Ergebnissen der Robotik steuert.

Der Kybernetiker Valentin Braitenberg hat das Buch I veicoli pensanti geschrieben. Wir nennen eine Formulierung wie «denkende Vehikel» einen Anthropomorphismus. Menschen, die allereinfachste fahrende Roboter (agents) sehen und verstehen, dass diese je nach Ausstattung von Sensorik und Motorik Verhaltensweisen zeigen, die wir «kennen» (wir nennen sie ängstlich, aggressiv, liebend oder neugierig), benutzen einen Anthropomorphismus. Wir vermuten ein absichtsvolles Verhalten, wenn wir zu sehen glauben, dass jedes Vehikel gewisse Zielpunkte zu erreichen und gewisse Situationen zu vermeiden bestrebt ist.

Für uns Aussenseiter sind solche Anthropomorphismen nicht einfach Missverständnisse, welche die Robotik-Forschung ans Licht bringt, sondern sie lassen sich auch als nützliche Heurismen verstehen. Wenn wir in Tiere, aber auch in Roboter mentale Zustände hineinlesen, kann das zunächst und vor allem ernüchternd wirken.

Wie solche Anthropomorphismen zustande kommen, verdeutlichen wir an einem Beispiel und erinnern mit Pfeifer/Bongard (2006: 36) zunächst an die bekannte Tatsache, dass Tiere, Menschen und Roboter Systeme sind, die über spezifische Sets von Sensoren verfügen, «die ständig sich verändernde Stimulationsmuster hervorrufen. Sie enthalten reiche Information über die realeWelt». Diese «teilt sich uns nicht über Etiketten oder Kategorien mit: Jeder muss selbst den Sinn dieser Sensorstimulation herausfinden». Roboter sind keine Computer der uns geläufigen Art, sondern zeigen analoge Eigenschaften zu Lebewesen: «Die wahrhaft autonomen Roboter, jene die weitgehend von menschlicher Kontrolle unabhängig sind, müssen situiert werden, d. h. sie müssen ihre Umgebung durch ihre sensorischen Systeme selber kennenlernen» (a.a.O. [2006: 36]). Just diese Befähigung zur Situierung schafft ein Anthropomorphismusproblem.

Die Robotik der Embodied Artificial Intelligence hat sich während einer ersten Phase vor allem nichtmenschlichen biologischen Systemen zugewandt: Insekten, Schlangen, Ratten. Um dem Leser eine Ahnung zu vermitteln, wie heute mit Robotern experimentiert wird, berichten wir von dem «Sahabot» genannten Roboter. In diesem Falle wurden der Bewegungsapparat, die Morphologie und die sensorischen Fähigkeiten der Wüstenameise Cataglyphis nachgebildet. Der so entstandene Roboter wurde in der Wüste getestet, wobei er, wie das Insekt, ohne landschaftliche Orientierungspunkte sein Nest nach der Nahrungssuche 
wieder finden musste. Es wäre nun möglich, diesen Roboter auf Erfolg zu trimmen, indem ihm ein Schnappschuss-Modell - eine Analogie zum Bild im Kopf der Ameise - implantiert würde. Der agent hält dabei seine Umgebung beim Verlassen des Nestes auf einem fotographischen Bild fest, um sich dann auf dem Rückweg daran zu orientieren. Der Sahabot dagegen wurde so konstruiert, dass die Frage beantwortet werden konnte, ob der agent den Navigationsmechanismus im Hier und Jetzt und in der Interaktion mit seiner Umgebung entwickeln kann oder ob es sich um eine angeborene Kompetenz handelt. Es zeigte sich im Laufe von Experimenten mit dem der Ameise nachgebauten Sahabot, dass er einen dem Schnappschuss ähnlichen Mechanismus selber erlernt. Dabei ergab sich aus der Implantierung verschiedener Lösungsansätze, dass sich die Ameise am Muster des polarisierten Lichtes am Himmel orientiert. Diese Fähigkeit verdankt sie spezialisierten Photorezeptoren und den zugehörigen Neuronen im visuellen Cortex, welche schliesslich den Schnappschuss auslösen. Der agent verfügt damit über einen an bestimmten Lichtemissionen orientierten Kompass (allozentrischer Bezugsrahmen) und ist zudem befähigt, den zurückgelegten Weg zu integrieren (egozentrischer Bezugsrahmen), beides zusammen erlaubt ihm, sein Nest wieder zu finden (Pfeifer/Bongard [2006:84 f.]; Möller: [2006], [2008: 1-43]).

Damit stiess man auch bei diesem Tier auf die sensorische Möglichkeit der Wahrnehmung von polarisiertem Licht, welche bei Menschen nicht bekannt ist. (Das Studium von Tieren mit anderen Sinnesrezeptoren und entsprechend anderem Verhalten erlaubt, den Menschen in seiner spezifischen Art zu erkennen).

Sahabot gehört zu den Biorobotern, aber auch zur developmental robotics, da er ein Schnappschussmodell entwickelt. Er zeigt eine Entwicklung auf, die auf der Interaktion zwischen System und non-sozialer Umgebung basiert. Von den Forschergruppen um Cynthia Breazeal, Kerstin Dautenhahn, Luc Steels u. a. werden demgegenüber Entwicklungsprozesse während sozial orientierten Interaktionen erforscht (Lungarella [2007]). Die Arbeit von Linda Smith und Cynthia Breazeal (2007) ist besonders interessant, wenn wir den Beitrag, den die Robotik für die Entwicklungspsychologie einmal leisten könnte, besser verstehen wollen.

Um dasWesentliche der menschlichen Intelligenz-im Sinne der Entwicklung von Überlebensstrategien in einer spezifischen Umgebung - zu erfassen, müsse man, so Smith und Breazeal, die für die Entwicklungsprozesse relevanten Prinzipien verstehen. Man habe Flugapparate aufgrund der Beobachtung von Vögeln konstruieren gelernt. Dabei seien nicht alle spezifischen Eigenschaften von Vögeln wie Federn, hohle Knochen, Flügelschlag, Schnäbel oder Eierlegen relevant gewesen. Relevant sei hingegen das Prinzip des aerodynamischen Auftriebs. Und so werde 
in der Artificial Intelligence nun auch nach den wesentlichen und mechanisch simulierbaren Prozessen des mentalen Wachstums geforscht. Man sei heute zwar weit entfernt davon, dieses Wachstum zu verstehen; doch haben sich bereits folgende Prinzipien als relevant herausgestellt:

Erstens: die Koordination von heterogenen Prozessen wie beispielsweise Hören, Sehen und Greifen etc. im Verlauf von konkreten Tätigkeiten.

Die Teilleistungen, die ein System, welches das soziale Interagieren erlernt, einbringen muss, sind:

, Die Fähigkeit des visuellen Nachverfolgens/Tracking von Gesichtsausdrücken oder Bewegungen eines Andern;

, die Möglichkeit, motorisch zu «plappern», das heisst, Gesichtsausdrücke oder andere Bewegungen ausgehend von einem elementaren Arsenal an Bewegungsmöglichkeiten ausprobieren und entwickeln zu können;

, Die Fähigkeit, die eigene Gesichtsstellung oder Bewegung wahrzunehmen;

, Die Fähigkeitzu einem Abgleich (mapping) von Regionen des eigenen Gesichts des Roboters mit jenem des menschlichen Gesichts («Organbeziehungen»);

Schliesslich muss das System über eine Kontingenzmetrik verfügen, die festhält, ob eine gesehene Bewegung vorübergehend kontingent ist mit der eigenen Bewegung.

Zweitens: Die Verbindung mit intelligenten Andern. Die Entwicklung finde immer unter Artgenossen mit ähnlichen inneren Systemen und ähnlichen äusseren Körpern statt.

In einem ihrer Experimente imitiert ein Mensch die Gesichtsausdrücke eines Roboters und der Roboter imitiert jene des Menschen. Die beiden Autorinnen schreiben: «Der Körper und seine Verhaltensweisen sind von Andern beobachtbar. Aber weil beobachtbares Verhalten auch verbunden (oder besser angetrieben) ist von inneren Zuständen, vermitteln beobachtbare körperliche Verhaltensweisen Andern auch - ungefähre - Informationen über diese inneren Zustände. Weil die eigenen Körperaktionen auch die inneren Zustände der Andern beeinflussen, sind die eigenen Aktionen auch (indirekt) verbunden mit den inneren Zuständen der Andern. Kurzum: Entwicklung geschieht in einem komplexen System verbundener Verhaltensweisen, verbundener Körper und verbundener kognitiver Systeme.» (Smith/Breazeal [2007:64], s. oben bez. Gegenübertragungsreaktion).

Dies ist eine Beschreibung wie sich eine reziproke Beziehung entwickelt. Und von hier ist es nicht mehr weit, dass die Robotiker ihren lernfähigen Systemen eine theory of mind zugestehen werden. 
Die beiden Autorinnen meinen dazu: «Die dynamische, sozial implementierte Verbindung zweier intelligenter [i.S. lernfähiger, R.S.] Systeme miteinander aufgrund ähnlicher Körper und Verhaltensweisen kann für eine wahrnehmende und sich bewegende Maschine - einen Roboter - tatsächlich genug sein, um sozial angepasste Antworten zu geben, welche Annahmen über den inneren Zustand von Andern reflektieren» (a.a.O.). Die Befähigung, Gesichtsausdrücke von Andern zu imitieren, könnte ein Mechanismus sein, der dazu dient, innerlich die Gefühle voneinander zu generieren.

Drittens: Wenn sich entwickelnde Organismen gleichzeitig verschiedene Aufgaben lernen, spricht man von überlappender Koordination. Als Beispiel dafür beschreiben die beiden Autorinnen die Rolle, welche die Koordination von Sehen und Hören für die Aufmerksamkeit spielt. Bei tauben Kindern entwickelt sich die visuelle Aufmerksamkeit ohne das Hören. Dabei legen Experimente und Beobachtungen nahe, dass während der Entwicklung der visuellen Aufmerksamkeit bei Blinden andere Teilprozesse ablaufen. So gibt es Bewegungen gegenüber eine grössere Empfindsamkeit, was wiederum eine grössere Ablenkbarkeit zur Folge hat. Allgemein lässt sich sagen, dass Entwicklung aus sich selbst heraus dank der Selbstorganisationstendenz geschieht, welche die Interaktion vieler Teilprozesse und Teilkomponenten mit sich bringt. So emergieren neue, komplexere kognitive Funktionen.

\section{$5 \quad$ Folgerungen}

In den vorangegangenen Abschnitten wurde in loser Reihung Material vorgestellt, an dem sich, wie wir meinen, für Psychoanalytiker das Weiterdenken der metapsychologischen Konzepte lohnt. Die Ausgangslage ist klar: Von unterschiedlichen Seiten wird gefragt, wie der Geist sich im Körper darstellt, welche Informationen der Körper abgibt, und in der Robotik wird versucht, örtlich situierte Körper in Bewegung unter real-time Bedingungen zu studieren.

Anthropomorphismen sind oft psychologischeVorstellungen, welche zusammen mit Kategorienfehlern als pseudotheoretische, die wirklichen Mechanismen und Prozesse verkennende Konstruktionen, die psychologische Theorie kompromittieren. Während das Anthropomorphisieren das Mentalisieren da kritisiert, wo es die Erkenntnis irreführt oder neue wissenschaftliche Erkenntnisse nicht aufgreift, erlaubt Kategoriendisziplin die genaue Reflexion und Kritik des Denkens und Beobachtens von andern. Und um dieses Mentalisieren ist es uns in diesem Aufsatz gegangen. 


\section{Literatur}

Beckermann, Ansgar (1977): Analytische Handlungstheorie II: Handlungserklärungen, Frankfurt: Suhrkamp.

Beckermann, Ansgar (2001): Analytische Einführung in die Philosophie des Geistes, Berlin/New York: de Gruyter.

Bilder, Robert M.; F., Frank LeFever (1998) Hrsg., Neuroscience of the Mind on the Centennial of Freud's Project for a Scientific Psychology. Annals of the New York Academy of Sciences 843.

Bolm, Thomas (2009): Mentalisierungsbasierte Therapie (MBT). Köln: Deutscher Ärzteverlag.

Leuzinger-Bohleber, Marianne (2009): Frühe Kindheit als Schicksal: Trauma, Embodiment, Soziale Desintegration. Psychoanalytische Perspektiven. Mit kinderanalytischen Fallberichten von Angelika Wolff und Rose Ahlheim, Stuttgart: Kohlhammer.

Leuzinger-Bohleber, Marianne und Rolf Pfeifer (2006). Recollecting the Past in the Present. Memory in the Dialogue between Psychoanalysis and Cognitive Science. In: Mauro Mancia, Hrsg., Psychoanalyis and Neuroscience. Milano: Springer, 63-97.

Leuzinger-Bohleber, Marianne; Tamara Fischmann (2006): What Is Conceptual Research in Psychoanalysis? In: Int J Psychoanal. 2006 October, 1355-86.

Lorenz, Kuno (1995): «Kategorienfehler», in: Jürgen Mittelstrass, Hrsg., Enzyklopädie Philosophie und Wissenschaftstheorie 2, Stuttgart/Weimar: Metzler, 370b-371a.

Lungarella, Max (2007): Developmental Robotics. In: Scholarpedia, Vol. 2, No. 8, 3104.

Meggle, Georg (1977): Analytische Handlungstheorie I: Handlungsbeschreibungen, Frankfurt: Suhrkamp.

Möller, Ralf (2006): Das Ameisenpatent. Bioroboter und ihre tierischen Vorbilder. Heidelberg: Spektrum.

Pauen, Michael; Gerhard Roth, Hrsg. (2001): Neurowissenschaften und Philosophie, München: W. Fink,-

Pfeifer, Rolf; Scheier, Christian (1999): Understanding Intelligence. Cambridge: M.I.T. press.

Pfeifer, Rolf; Josh Bongard (2006): How the Body Shapes the Way We Think. Cambridge: MIT press. 
Pöppel, Ernst, Eva Ruhnau (2010): Psychologie als eine auf Modelle angewiesene Angelegenheit ohne Taxonomie - eine Polemik, http://www.ernst-poeppel. de/Psychologie-als-eine-auf-Modelle---.

Potthoff, Peter (2008 [2010]): Mentalisierung und gruppenanalytische Behandlungstechnik, in: Mathias Hirsch, Hrsg., Die Gruppe als Container. Mentalisierung und Symbolisierung in der analytischen Gruppenpsychotherapie, Göttingen: Vandenhoeck \& Ruprecht, 86-116.

Schafer, Roy (1982): Eine neue Sprache für die Psychoanalyse, Stuttgart: KlettCotta.

Schmidt-Hellerau, Cordelia (2003): Lebenstrieb \& Todestrieb; Libido \& Lethe. Ein formalisiertes konsistentes Modell der psychoanalytischen Trieb- und Strukturtheorie. Giessen: Psychosozial-Verlag.

Smith, Linda B.; Cynthia Breazeal (2007): The Dynamic Lift of Developmental Process. In: Developmental Science 10:1, 61-68.

Tucker, Don M. (2007): Mind from Body. Experience from Neural Structure. Oxford: Univ. Press.

Tucker, Don M.; Phan Luu (1998): Cathexis Revisted: Corticolimbic Resonance and the Adaptive Control of Memory. In: R. Bilder, F. LeFever (1998:134-152).

Winnicott, Donald Woods (1971): Vom Spiel zur Kreativität, Stuttgart: Klett-Cotta 122010 . 